\title{
Pathogenesis of Helicobacter pylori infection
}

Vania Camilo ${ }^{1,2}$, Toshiro Sugiyama ${ }^{3 *}$ and Eliette Touati ${ }^{{ }^{*}}$

${ }^{1}$ Institut Pasteur, Département de Microbiologie, Unité de Pathogenèse de Helicobacter, ERL CNRS 3526, 75724 Paris cedex 15, France. ${ }^{2}$ INSERM U1173, Faculty of Health Sciences Simone Veil, Université Versailles-Saint-Quentin, 78180 Saint Quentin en Yvelines, France. ${ }^{3}$ University of Toyama, Graduate School of Medicine and Pharmaceutical Sciences, Department of Gastroenterology, 2630 Sugitani, Toyama, Japan

Correspondance*

Eliette Touati, Institut Pasteur, Unité de Pathogenèse de Helicobacter, 28 rue du Dr Roux, 75724 Paris cedex 15, France. Phone: +33 14061 37 85; Fax: +33 140613640

Email: eliette.touati@pasteur.fr

Toshiro Sugiyama, University of Toyama, Graduate School of Medicine and Pharmaceutical Sciences, Department of Gastroenterology, 2630 Sugitani, Toyama, Japan.

Phone: 81-76-434-7300; Fax: 81-76-434-5027

Email: tsugi@med.u-toyama.ac.jp 


\begin{abstract}
Helicobacter pylori is responsible for the most commonly found infection in the World's population. It is the major risk factor for gastric cancer development. Numerous studies published over the last two years provide new insights into the strategies employed by $H$. pylori to adapt to the extreme acidic conditions of the gastric environment, to establish persistent infection and to deregulate host functions, leading to gastric pathogenesis and cancer. In this review, we report recent data on the mechanisms involved in chemotaxis, on the essential role of nickel in acid-resistance and gastric colonization, on the importance of adhesins and Hop proteins and on the role of CagPAI-components and CagA. Among the host functions, the escape of immune response, the ability of bacteria to induce genetic instability and modulate telomeres, the mechanism of autophagy and the deregulation of micro RNAs are specially focused.
\end{abstract}

Key words: Acid-resistance, Chemotaxis, Adhesins, nickel, CagA, Type IV secretion system, VacA, Evasion, autophagy, microRNA 


\section{H. pylori gastric colonization and acid-resistance}

\section{Motility}

A specific characteristic of $H$. pylori is its ability to resist to the extreme acidic conditions of the stomach. Flagella are essential for bacteria swimming, to reach the protective mucus layer at the surface of the gastric mucosa. The ferric uptake regulator Fur which regulates iron homeostasis, acid acclimation and oxidative response has been shown to be important for $H$. pylori colonization. Recently, Lee et al. reported that Fur positively regulates the flagellar motor switch in the $H$. pylori strain J99. ${ }^{1}$ The enzyme LuxS plays an essential role in $H$. pylori motility. It catalyzes the production of the autoiducer-2 (AI-2) which is decreased in the fur mutant, thus indicating that Fur also controls $H$. pylori motility through its action on AI-2 production. Another H. pylori factor recently reported to modulate motility is HP0231 which is a Dsb-like protein. HP0231 participates in redox homeostasis and is essential for gastric colonization. Its lack also impairs type IV secretion system and VacA functions. ${ }^{2}$

\section{Chemotaxis}

H. pylori has four chemoreceptors TlpA,B,C,D, a CheA kinase, a CheY responsive regulator and multiple coupling proteins, essential for chemotaxis. The combination of $\mathrm{CheW}$ and $\mathrm{CheV} 1$ results in proper formation of the CheA chemotaxis complex and activation of the CheA kinase activity, optimizing chemotaxis function. ${ }^{3}$ CheA is repressed by the histone-like (HU) protein in response to acid stress. ${ }^{4}$ CheA is the sensor of the two-component system CheA-CheY that regulates the movement of flagella according to environmental changes, as higher $\mathrm{H}^{+}$ions concentration. Huang et al. reported a new and independent role for two chemoreceptors as acid sensors. ${ }^{5}$ Using a videomicroscopy method, they showed that $H$. pylori mutants lacking $\mathrm{TlpB}(\Delta t \mathrm{tpB})$ are able to detect and to swim away from an $\mathrm{HCl}$ gradient. In addition, they identified TlpD as an independent acid sensor. The inactivation of 
both TlpA and TlpD jointly decreased gastric colonization in mice. In an independent study, authors showed that TlpD localization and TlpD-dependent energy sensing of H. pylori, which is likely to also mediate acid repellent sensing, are modulated through protein-protein interactions involving the chemotaxis histidine kinase CheAY2, the central metabolic enzyme aconitase AcnB and the anti-oxidant enzyme catalase KatA. ${ }^{6}$ In addition to oxidative stress, iron depletion and iron-sulfur repair system NifSU also alter TlpD-dependent bacteria behaviour. Furthermore, a separate study, performing genome-wide and chromatinimmunoprecitation analysis (ChIP), identified $\operatorname{tlp} B$ as a target for the orphan response regulator HP1043 (HsrA), which has been proposed to modulate the response to environmental conditions associated to stress signals and protein synthesis arrest. ${ }^{7}$

\section{Nickel is essential for gastric colonization by $\mathrm{H}$. pylori.}

An optimal balance between nickel uptake and incorporation in metalloenzymes is essential for gastric colonization by $H$. pylori. Nickel binding is required at the $\mathrm{N}$-terminal NHE motif of the Ni-metallochaperone HypA, involved in urease and [NiFe] hydrogenase maturation which are essential for acid survival of $H$. pylori. ${ }^{8}$ Fischer et al. identified a novel H. pylori nickel transport system NiuBDE. ${ }^{9}$ NiuBDE is required for nickel-dependent urease activation and acid survival. It is essential for gastric colonization. This transport system is specific for gastric Helicobacter species. In addition, a non-toxic intracellular level of nickel is maintained by two small nickel binding histidine-rich paralogs, Hpn and Hpn-2, which are also essential for gastric colonization. ${ }^{10}$ The acquisition of Hpn and Hpn-2 is specific to gastric Helicobacter species.

Nickel is a cofactor for NikR, a transcriptional regulator of nickel-responsive genes. By combining RNA and ChIP sequencing methodologies, Vanini et al. identified NikR-target genes including metal-ions transporters, virulence factors, non-coding RNAs and toxinantitoxin systems, thus reflecting a pleiotropic adaptation to stresses. ${ }^{11} \mathrm{NikR}$ is also a positive 
transcriptional regulator of ArsRS involved in acid adaptation. Roncarati et al. ${ }^{12}$ reported a metal-responsive promoter DNA compaction mechanism which modulates the transcriptional regulation and coordinated response to environmental stimuli. In the presence of nickel, NikR antagonizes the Fur-mediated regulation and DNA compaction at the ArsRS promoter.

\section{Adhesion and outer membrane proteins}

The blood group antigen binding adhesin (BabA) is the best-studied adhesin of H. pylori. It binds to $\mathrm{ABO} / \mathrm{Le}^{\mathrm{b}}$ blood group antigens (Leb), fucosylated carbohydrates expressed by gastric epithelial cells and mucins. H. pylori BabA-mediated binding has been recently reported to be acid sensitive, responsive to and reversible by increasing $\mathrm{pH} .{ }^{13}$ Interestingly, the BabA protein sequence impacts acid sensitivity in Leb binding and plays an essential role in acidadaptation of bacteria in response to variation of acid secretion during disease progression. Binding of $\mathrm{BabA}$ to $\mathrm{Le}^{\mathrm{b}}$-glycoconjugates inhibits bacteria proliferation due to formation of bacterial aggregates, suggesting a new role of mucins in the host defence against $H$. pylori. ${ }^{14}$

In animal models, expression of $b a b A$ is lost either by phase variation or gene conversion with recombination of the $b a b B$ paralog into the $b a b A$ locus. By comparing the response of rhesus macaques to different isogenic $b a b A$ or $b a b B$ mutants, Hansen et al. showed an independent selective pressure for loss of BabA expression and overexpression of BabB. ${ }^{15}$ In addition, loss of BabA expression was reported to be independent of adaptive immunity or Toll like receptor signaling. ${ }^{16}$

A novel interaction between bacterial adhesin HopQ and carcinoembryonic antigen-related cell adhesion molecules (CEACAMs) family members was recently described and combined with structural information about HopQ. ${ }^{17}$ This interaction was reported to be largely CEACAM glycosylation-independent, mediated through the IgV-like N-terminal domains of CEACAMs and a $\beta$-hairpin insertion in HopQ's extracellular $3+4$ helix bundle domain. It seems to be relevant for CagA translocation into host cells and provides a mechanistic 
explanation for the binding of $H$. pylori to the gastric epithelium of non-secretors. ${ }^{18}$ Interestingly, CEACAM-6 was found to be upregulated by CagA and may represent a diagnostic biomarker of early gastric cancer. ${ }^{19}$

\section{Components of the Cag pathogenicity island}

The virulence of $H$. pylori is intimately associated with the presence of a cag pathogenicity island (cagPAI) locus that encodes for a type IV secretion system (T4SS) and the bacterial oncoprotein, CagA.

\section{T4SS components}

Loss of function of the T4SS during in vivo adaptation in mice and primate models is frequent and has been associated to recombination in cagY. Suarez et al., demonstrated that upon chronic H. pylori infection, cagY recombination could modulate T4SS function in humans and rodents, which may be important for a successful long-term persistence of infection. The mechanism of $\operatorname{cag} Y$ alterations was different in human and gerbil isolates, with predominance of single base pair mutations in human samples and addition/deletion of motifs in the gerbil reisolates. ${ }^{20}$ These $c a g Y$ recombination events may represent a means to evade the host immune response since they were not found in the Rag $1^{-/-}$immunodeficient mouse model. ${ }^{21}$ Two additional mouse models, with deletion of the interferon gamma receptor (IFNGR) and IL10 ${ }^{-/}$, have further demonstrated that a CD4 ${ }^{+}$, Th1-polarized response is required for the CagY-mediated T4SS loss of function. ${ }^{22}$ H. pylori pathogenicity also depends on other components of the T4SS, such as CagL, CagI and CagU. CagL is found at the tip of T4SS where its interaction through an arginine-glycine-aspartate (RGD) motif with host's $\alpha 5 \beta 1$ integrin is essential for CagA translocation. Recently, two novel integrin-binding motifs, TSPSA and TASLI, the latter one located opposite the RGD motif, were described. ${ }^{23}$ In 
addition, the C-terminal coiled-coil region of CagL seems to be essential for adhesion and IL8 production in a $\beta 1$-integrin/FAK-independent and TGF $\alpha /$ EGFR-dependent manner. ${ }^{24} \mathrm{CagI}$ is also a $\beta 1$ integrin-binding component of the T4SS that may bind and stabilize CagA. ${ }^{25}$ The relevance of $\mathrm{CagU}$ for the function and biogenesis of T4SS was also recently revealed. CagU is an inner membrane-associated protein, partially processed at C-terminal and presumably able to self-associate into dimers. Importantly, CagA surface localization seems to be CagUdependent. ${ }^{26}$ In addition to proteins encoded within the cagPAI, the autotransporter ImaA (HP0289) has been recently reported to modulate the initial H. pylori- $\alpha 5 \beta 1$ integrin interaction, preceding the cagPAI T4SS-interactions and associated pro-inflammatory activities. ${ }^{27}$ ImaA may also directly affect the level of host integrin.

\section{CagA and VacA virulence factors}

H. pylori colonization is dependent of the successful manipulation of the host's cells that is detrimental for the host in the long term. Polymorphisms in the CagA multimerization motif (CM) were described among geographic variants of CagA, namely the East Asian and Western strains. ${ }^{28}$ These polymorphisms condition CagA's binding to and inhibition of PAR1b, a protein essential for the establishment and maintenance of cell polarity. Disruption of cell polarity by PAR1b inhibition promotes transcytosis of iron to the basolateral side of the host cell, therefore contributing to successful colonization of the gastric niche. Furthermore, Jang et al., showed that some H. pylori strains are heterogeneous in terms of cagA copies (between 0 and 4 copies), arranged in tandem within the chromosome. The number of $\operatorname{cag} A$ copies can change dynamically and is directly associated with toxicity. ${ }^{29}$ After delivery via T4SS, CagA becomes phosphorylated by members of the Src and Abl family kinases at EPIYA (Glu-Pro-Ile-Tyr-Ala) motifs present at its C-terminus. This phosphorylation allows binding to $\mathrm{SH} 2$ domain-containing proteins, such as SHP2 tyrosine phosphatase. Saju et al., reported that SHP2 homologue, SHP1, can bind to CagA in an 
EPIYA-independent way and lessen its oncogenic effect by promoting dephosphorylation of EPIYA motifs in CagA. Interestingly, Epstein-Barr Virus (EBV) can induce SHP1 promoter hypermethylation, both in vitro and in vivo, hence providing a mechanistic link between EBV and H. pylori co-infection and gastric cancer development. ${ }^{30}$

Since CagA is associated with $H$. pylori-induced MALT lymphoma and since the infiltrating lymphocytes in the gastric mucosa can contact directly with $H$. pylori, Krish et al., used B-cell chronic lymphocytic leukemia derived cells (MEC1 cells) as an infection model. ${ }^{31}$ They observed that CagA was rapidly injected and tyrosine phosphorylated by cell kinases of the Src and Abl families. This implies that inhibitors of these kinases could be useful for the treatment of H. pylori-associated MALT lymphoma.

VacA (vacuolating cytotoxin A) is another virulence factor of $H$. pylori strains. The VacA cytotoxicity is determined by allelic diversity within the signal region (s1/s2), middle region $(\mathrm{m} 1 / \mathrm{m} 2)$ and intermediate region (i1/i2). Sinnet et al. ${ }^{32}$, described a new polymorphism within the 5'UTR of vacA mRNA that significantly associated with gastric inflammation levels of $H$. pylori positive patients and stratified disease risk among vacA il-type strains. Another study reported a VacA-dependent pathway leading to CagA phosphorylation in a duodenum carcinoma cell line, AZ-521. VacA induced phosphorylation of Src kinase at Tyr418 via its receptor RPTP $\alpha$. This in turn led to phosphorylation of CagA at Tyr972 by the activated Src and enabled pCagA association with SHP2 phosphatase. This VacA effect on Src seems to be cell line specific. ${ }^{33}$

\section{Deregulation of host functions}

\section{Immune Escape Mechanisms and Deregulation of Host Cells}

The majority of the H. pylori-infected individuals are asymptomatic and only one proportion will develop gastric or duodenal ulcer, gastric mucosa-associated lymphoid tissue 
lymphoma or gastric cancer during long-term infection. Hence, the precise understanding of the molecular mechanisms regarding host responses to $H$. pylori might allow a more effective intervention.

Once translocated into the cells, the CagA oncoprotein activates various intracellular signalling pathways, including NF- $\mathrm{B}$ pathway and MAP kinases via EGFR activation. ${ }^{34}$ Previous papers reported that H. pylori mainly induced transactivation of EGFR via heparinbinding EGF-like growth factor (HB-EGF) that was released from gastric epithelial cells. Recently, Zaidi et al. demonstrated that a novel serine residue phosphorylation of EGFR leads to the internalization and activation of $\mathrm{EGFR}^{35}$, independently of HB-EGF binding. At an early stage of $H$. pylori infection, this novel EGFR activation pathway is essential for production of human $\beta$-defensin 3 (hBD3), the most potent member of anti-microbial peptides (AMPs) family. The members of the AMP family (hBD1, hBD2, hBD3) have been extensively investigated as an important host defence against $H$. pylori infection. hBD1 is constitutively expressed in uninfected gastric epithelial cells, and it is downregulated in gastric epithelial cells with $H$. pylori infection. ${ }^{36}$ Expression of hBD2 is dependent on the presence of $H$. pylori cagPAI and modulated by nucleotide-binding oligomerization domain-1 (NOD1). ${ }^{37}$ Finally, expression of hBD3 is dependent on the EGFR/MAP kinase pathway. Importantly, hBD3 exerts a 100 times more potent anti- $H$. pylori activity than hBD2, in in vitro assays. ${ }^{38}$ Therefore, hBD3 release from $H$. pylori-infected gastric cells is crucial for a first-line host innate immune response. Muhammad et al. demonstrated that, in the early phase of infection, TAK1-p38 $\alpha$ pathway mediated the phosphorylation the aforementioned EGFR serine residue, which then induced the production of hBD3 via MAP kinase and JAK/STAT signalling, in AGS cells. Interestingly, in this study, infection with a CagA knockout H. pylori strain did not inhibit the downregulation of hBD3 in late phase. ${ }^{39}$ This is in contrast with a previous report demonstrating that, in the late phase of co-culture, hBD3 was downregulated 
by the $\mathrm{CagA}^{38}$ via SHP-2, therefore terminating EGFR activation and its downstream signalling. Nevertheless, the inevitable limitation of these reports is that in vitro experimental evidence cannot exclude the interaction among several intracellular signalling molecules, or the modifications introduced from interactions with immune cells during chronic persistent infection.

\section{DNA damage and Telomere length}

H. pylori infection-induced DNA damage in the epithelial cells is a major contributing factor to the development of gastric cancer. Previous publications demonstrated that $H$. pylori infection introduced DNA double-strand breaks (DSBs) in several gastric carcinogenesis models, such as transgenic insulin-gastrin (INS-GAS) mice and mongolian gerbils, by promoting an inflammatory response and by inducing epithelial DNA damage. Sierra et al. showed that gefitinib, a specific EGFR inhibitor, was able to decrease the epithelial DNA damage induced by $H$. pylori infection of C57BL/6 mice. Furthermore, it blocked H. pyloriinduced activation of MAPK1/3 and AP-1 in gastric epithelial cells and markedly reduced dysplasia and carcinoma in infected INS-GAS mice and gerbils. ${ }^{40}$ This suggests that in $H$. pylori infected individuals, epithelial EGFR blockage may represent a putative cancer preventive strategy.

Telomere shortening in genomic DNA is an indirect footprint, which reflects an accumulation of oxidative stress and chromosomal instability as a result of life-long infection. Tahara et al. demonstrated that H. pylori- infected gastric cancer tissues and the adjacent noncancer tissues possessed shorter telomere length than $H$. pylori-negative gastric tissues and that the shortening of telomere length was associated with higher expression of $I L-1 B$ and $N F$ $k B .{ }^{41}$

\section{H. pylori infection and Autophagy}


Autophagy is a homeostatic process involving sequestration of cytoplasmic components in autophagosomes, which leads to their degradation and recycling. Autophagy has been involved in limiting inflammation, tissue damage, and genomic instability. Therefore, autophagy impairment by $H$. pylori infection is considered to be an important early step in gastric carcinogenesis. ${ }^{42}$

Tanaka et al.'s microarray analysis of 266 autophagy-related genes (ATG) revealed that 16 genes were upregulated and 9 genes were downregulated in $H$. pylori-infected gastric mucosa. ${ }^{43}$ ATG16L1 mRNA levels were inversely correlated with $H$. pylori density as well as gastric atrophy in gastric mucosa. Furthermore, an $A T G 16 L 1$ polymorphism seems to be determinant for susceptibility to $H$. pylori infection.

MAPILC3A (microtubule-associated protein 1 light chain 3; one ATG with CpG islands) is a major regulator of autophagosome formation. It is implicated in the conversion of the cytosolic truncated form of LC3 (LC3-I) to an autophagosomal membrane-associated, phosphatidylethanolamine-conjugated form (LC3-II), an indicator of autophagosome formation. Muhammad et al. demonstrated that MAP1LC3A variant1 (MAP1LC3Av1) was methylation-silenced in $H$. pylori-infected gastric cancer tissues as well as adjacent noncancer tissues, but not in $H$. pylori negative gastric tissues. In addition, cells with $M A P 1 L C 3 A v$ knock-down displayed more proliferative and more invasive characteristics in in vitro studies. Therefore, the inactivation of MAP1LC3Av1, which disrupts the autophagy pathway, may contribute to carcinogenesis in gastric epithelial cells. ${ }^{44}$

\section{MicroRNAs and Deregulation of Host Cells}

MicroRNAs (miRNAs) are post-transcriptional regulators of gene expression involved in development, cellular proliferation and oncogenesis. Some miRNAs are altered in cancer cells and function either as oncogenes or tumor suppressor genes. 
Noormohammad et al. revealed that miR-222 is an onco-miRNA that is upregulated in gastric cancer tissues compared with normal tissues. However, this upregulation seems to be independent of $H$. pylori. ${ }^{45}$ Wang et al. compared the miRNA expression in H. pylori $(+)$ gastric cancer and H. pylori(-) gastric cancer. Among 53 different mRNAs, the expression of miR-143-3p was the most significantly upregulated in $H$. pylori $(+)$ gastric cancer tissues. In vitro assays revealed a negative regulation on cell growth, apoptosis, invasion and migration, showing that miR-143-3p acts as tumour suppressor miRNA by targeting AKT2. ${ }^{46}$ Regarding H. pylori-related virulence factors, Zou et al. demonstrated that Tip- $\alpha$, a carcinogenic factor of H. pylori, activated NF- $\kappa \mathrm{B}$ by inhibiting the miR-3178-mediated targeting of TRAF3. ${ }^{47} \mathrm{MiR}-$ 124 has tumour suppressive functions and is regulated by $\mathrm{CpG}$ methylation silencing. ${ }^{48}$ Murray-Stewart et al. reported that miR-124 is a negative regulator of polyamine catabolic enzyme spermine oxidase (SMOX), which is induced in H. pylori gastritis. ${ }^{49}$ Reporter assays demonstrated that a direct interaction of miR-124 with the 3'-UTR of SMOX mRNA is linked to this negative regulation. These results suggested a protective role for miR-124 through the inhibition of SMOX-mediated DNA damage in H. pylori $(+)$ gastric cancer.

\section{Acknowledgements}

We sincerely apologize to all authors who have published in the field on Helicobacter pathogenesis during the past two years that we could not cite in this review due to length limitations. VC is financially supported by the ANR 15-CE17-0015-05 EMMIE project.

\section{Conflicts of interest}

The authors have declared no conflicts of interest 


\section{References}

1. Lee AY, Kao CY, Wang YK, et al. Inactivation of ferric uptake regulator (Fur) attenuates Helicobacter pylori J99 motility by disturbing the flagellar motor switch and autoinducer-2 production. Helicobacter. 2017. Apr 12. doi: 10.1111/hel.12388

2. Zhong Y, Anderl F, Kruse T, et al. Helicobacter pylori HP0231 influences bacterial virulence and is essential for gastric colonization. PLoS One. 2016;11:e0154643.

3. Abedrabbo S, Castellon J, Collins KD, et al. Cooperation of two distinct coupling proteins creates chemosensory network connections. Proc Natl Acad Sci USA. 2017;114:2970-2975.

4. Alvarez A, Toledo $\mathrm{H}$. The histone-like protein $\mathrm{HU}$ has a role in gene expression during the acid adaptation response in Helicobacter pylori. Helicobacter. 2017. Feb 28. doi: 10.1111/hel.12381.

5. Huang JY, Goers Sweeney E, Guillemin K, et al. Multiple Acid Sensors Control Helicobacter pylori colonization of the stomach. PLoS Pathog. 2017;13:e1006118.

6. Behrens W, Schweinitzer T, McMurry JL, et al. Localisation and protein-protein interactions of the Helicobacter pylori taxis sensor TlpD and their connection to metabolic functions. Sci Rep. 2016;6:23582.

7. Pelliciari S, Pinatel E, Vannini A, et al. Insight into the essential role of the Helicobacter pylori HP1043 orphan response regulator: genome-wide identification and characterization of the DNA-binding sites. Sci Rep. 2017;7:41063.

8. Hu HQ, Johnson RC, Merrell DS, et al. Nickel ligation of the N-terminal amine of HypA is required for urease maturation in Helicobacter pylori. Biochemistry. 2017;56:1105-1116.

9. Fischer F, Robbe-Saule M, Turlin E, et al. Characterization in Helicobacter pylori of a nickel transporter essential for colonization that was acquired during evolution by gastric Helicobacter species. PLoS Pathog. 2016;12:e1006018.

10. Vinella D, Fischer F, Vorontsov E, et al. Evolution of Helicobacter: acquisition by gastric species of two histidine-rich proteins essential for colonization. PLoS Pathog. 2015;11:e1005312.

11. Vannini A, Pinatel E, Costantini PE, et al. Comprehensive mapping of the Helicobacter pylori NikR regulon provides new insights in bacterial nickel responses. Sci Rep. 2017;7:45458.

12. Roncarati D, Pelliciari S, Doniselli N, et al. Metal-responsive promoter DNA compaction by the ferric uptake regulator. Nat Commun. 2016;7:12593.

13. Bugaytsova JA, Bjornham O, Chernov YA, et al. Helicobacter pylori adapts to chronic infection and gastric disease via pH-responsive BabA-mediated adherence. Cell Host Microbe. 2017;21:376-389.

14. Skoog EC, Padra M, Aberg A, et al. BabA dependent binding of Helicobacter pylori to human gastric mucins cause aggregation that inhibits proliferation and is regulated via ArsS. Sci Rep. 2017;7:40656.

15. Hansen LM, Gideonsson P, Canfield DR, et al. Dynamic Expression of the BabA Adhesin and its BabB Paralog During Helicobacter pylori Infection in Rhesus Macaques. Infect Immun. 2017. Apr 10. doi: 10.1128/IAI.00094-17.

16. Kable ME, Hansen LM, Styer CM, et al. Host Determinants of Expression of the Helicobacter pylori BabA Adhesin. Sci Rep. 2017;7:46499.

17. Koniger V, Holsten L, Harrison U, et al. Helicobacter pylori exploits human CEACAMs via HopQ for adherence and translocation of CagA. Nat Microbiol. 2016;2:16188. 
18. Javaheri A, Kruse T, Moonens K, et al. Helicobacter pylori adhesin HopQ engages in a virulence-enhancing interaction with human CEACAMs. Nat Microbiol. 2016;2:16189.

19. Roy RK, Hoppe MM, Srivastava S, et al. CEACAM6 is upregulated by Helicobacter pylori CagA and is a biomarker for early gastric cancer. Oncotarget. 2016;7:5529055301.

20. Suarez G, Romero-Gallo J, Sierra JC, et al. Genetic manipulation of Helicobacter pylori virulence function by host carcinogenic phenotypes. Cancer Res. 2017;77:2401-2412.

21. Barrozo RM, Cooke CL, Hansen LM, et al. Functional plasticity in the type IV secretion system of Helicobacter pylori. PLoS Pathog. 2013;9:e1003189.

22. Barrozo RM, Hansen LM, Lam AM, et al. CagY Is an Immune-Sensitive Regulator of the Helicobacter pylori Type IV Secretion System. Gastroenterology. 2016;151:11641175.e3.

23. Bonig T, Olbermann P, Bats $\mathrm{SH}$, et al. Systematic site-directed mutagenesis of the Helicobacter pylori CagL protein of the Cag type IV secretion system identifies novel functional domains. Sci Rep. 2016;6:38101.

24. Wiedemann T, Hofbaur S, Loell E, et al. A C-Terminal Coiled-Coil Region of CagL is Responsible for Helicobacter pylori-Induced Il-8 Expression. Eur J Microbiol Immunol (Bp). 2016;6:186-196.

25. Wang H, Yao Y, Ni B, et al. Helicobacter pylori CagI is associated with the stability of CagA. Microb Pathog. 2016;99:130-134.

26. Kumari R, Shariq M, Kumar N, et al. Biochemical characterization of the Helicobacter pylori Cag-type IV secretion system unique component CagU. FEBS Lett 2017;591:500-512.

27. Sause WE, Keilberg D, Aboulhouda S, et al. The Helicobacter pylori autotransporter ImaA tempers the bacterium's interaction with alpha5betal integrin. Infect Immun. 2017; doi: 10.1128/IAI.00450-16

28. Nishikawa H, Hayashi T, Arisaka F, et al. Impact of structural polymorphism for the Helicobacter pylori CagA oncoprotein on binding to polarity-regulating kinase PAR1b. Sci Rep. 2016;6:30031.

29. Jang S, Su H, Blum FC, et al. Dynamic expansion and contraction of CagA copy number in Helicobacter pylori impact development of gastric disease. MBio. 2017; Feb 21;8(1) doi: 10.1128/mBio.01779-16.

30. Saju P, Murata-Kamiya N, Hayashi T, et al. Host SHP1 phosphatase antagonizes Helicobacter pylori CagA and can be downregulated by Epstein-Barr virus. Nat Microbiol. 2016;1:16026.

31. Krisch LM, Posselt G, Hammerl P, et al. CagA phosphorylation in Helicobacter pylori-infected B cells is mediated by the nonreceptor tyrosine kinases of the Src and Abl families. Infect Immun. 2016;84:2671-80.

32. Sinnett CG, Letley DP, Narayanan GL, et al. Helicobacter pylori vacA transcription is genetically-determined and stratifies the level of human gastric inflammation and atrophy. J Clin Pathol. 2016. doi: 10.1136/jclinpath-2016-203641

33. Nakano M, Yahiro K, Yamasaki E, et al. Helicobacter pylori VacA, acting through receptor protein tyrosine phosphatase alpha, is crucial for CagA phosphorylation in human duodenum carcinoma cell line AZ-521. Dis Model Mech. 2016;9:1473-1481.

34. Chaturvedi R, Asim M, Piazuelo MB, et al. Activation of EGFR and ERBB2 by Helicobacter pylori results in survival of gastric epithelial cells with DNA damage. Gastroenterology. 2014;146:1739-51.e14. 
35. Zaidi SF, Refaat A, Zhou Y, et al. Helicobacter pylori induces serine phosphorylation of EGFR via novel TAK1-p38 activation pathway in an HB-EGF-Independent manner. Helicobacter. 2015;20:381-9.

36. Patel SR, Smith K, Letley DP, et al. Helicobacter pylori downregulates expression of human beta-defensin 1 in the gastric mucosa in a type IV secretion-dependent fashion. Cell Microbiol. 2013;15:2080-92.

37. Grubman A, Kaparakis M, Viala J, et al. The innate immune molecule, NOD1, regulates direct killing of Helicobacter pylori by antimicrobial peptides. Cell Microbiol. 2010;12:626-39.

38. Bauer B, Pang E, Holland C, et al. The Helicobacter pylori virulence effector CagA abrogates human beta-defensin 3 expression via inactivation of EGFR signaling. Cell Host Microbe. 2012;11:576-86.

39. Muhammad JS, Zaidi SF, Zhou Y, et al. Novel epidermal growth factor receptor pathway mediates release of human beta-defensin 3 from Helicobacter pylori-infected gastric epithelial cells. Pathog Dis. 2016;74. 74(3). pii: ftv128.

40. Sierra JC, Asim M, Verriere TG, et al. Epidermal growth factor receptor inhibition downregulates Helicobacter pylori-induced epithelial inflammatory responses, DNA damage and gastric carcinogenesis. Gut. 2017;35:5480-5488.

41. Tahara T, Tahara S, Tuskamoto T, et al. Telomere length in the gastric mucosa after Helicobacter pylori eradication and its potential role in the gastric carcinogenesis. Clin Exp Med. 2017;Feb 14. doi: 10.1007/s10238-017-0458-1.

42. Raju D, Hussey S, Ang M, et al. Vacuolating cytotoxin and variants in Atg16L1 that disrupt autophagy promote Helicobacter pylori infection in humans. Gastroenterology. 2012;142:1160-71.

43. Tanaka S, Nagashima H, Uotani T, et al. Autophagy-related genes in Helicobacter pylori infection. Helicobacter 2017; Jun;22(3). doi: 10.1111/hel.12376.

44. Muhammad JS, Nanjo S, Ando T, et al. Autophagy impairment by Helicobacter pylori-induced methylation silencing of MAP1LC3Av1 promotes gastric carcinogenesis. Int J Cancer. 2017;140:2272-2283.

45. Noormohammad M, Sadeghi S, Tabatabaeian H, et al. Upregulation of miR-222 in both Helicobacter pylori-infected and noninfected gastric cancer patients. $J$ Genet. 2016;95:991-995.

46. Wang F, Liu J, Zou Y, et al. MicroRNA-143-3p, up-regulated in H. pylori-positive gastric cancer, suppresses tumor growth, migration and invasion by directly targeting AKT2. Oncotarget. 2017;8:28711-28724.

47. Zou M, Wang F, Jiang A, et al. MicroRNA-3178 ameliorates inflammation and gastric carcinogenesis promoted by Helicobacter pylori new toxin, Tip-alpha, by targeting TRAF3. Helicobacter. 2017;Apr;22(2). doi: 10.1111/hel.12348.

48. Ando T, Yoshida T, Enomoto S, et al. DNA methylation of microRNA genes in gastric mucosae of gastric cancer patients: its possible involvement in the formation of epigenetic field defect. Int J Cancer. 2009;124:2367-74.

49. Murray-Stewart T, Sierra JC, Piazuelo MB, et al. Epigenetic silencing of miR-124 prevents spermine oxidase regulation: implications for Helicobacter pylori-induced gastric cancer. Oncogene. 2016;35:5480-5488. 